\title{
Corrigendum: Infrared light excites cells by changing their electrical capacitance
}

\author{
Mikhail G. Shapiro, Kazuaki Homma, Sebastian Villarreal, Claus-Peter Richter \& Francisco Bezanilla
}

Nature Communications 3:736 doi: 10.1038/ncomms1742 (2012); Published 13 Mar 2012; Updated 10 Nov 2017

We have been alerted that the theoretical explanation proposed in the subheading 'Capacitive effect is consistent with classical theory' of the Results section, to explain the molecular events underlying the experimentally determined increase in cell membrane electrical capacitance by infrared light excitation, is not valid. This has been attributed to an error in the assignment of the sign convention of the transmembrane charge model used in the proposed theoretical explanation. This error does not invalidate the experimental findings and main conclusion of the work, namely that membrane capacitance changes underlie infrared light-induced electrical currents and action potentials.

We thank Plaksin et al. for bringing this to our attention and direct readers to the Correspondence and our Reply, which details the exact nature of the error and includes an alternative explanation for the molecular mechanisms causing this experimental effect.

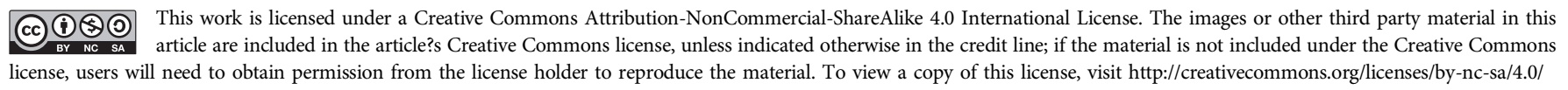

(C) The Author(s) 2017 